$\xi=$

\title{
The perceptions of patients on antiretroviral therapy (ART) and treatment supporters with regard to their role towards ART adherence, at ART clinics in the intermediate hospital Oshakati, Namibia
}

\author{
Olivia Ningeninawa Tuhadeleni ${ }^{1}$, Linda Ndeshipandula Nghipondoka- Lukolo ${ }^{2} *$ \\ ${ }^{1}$ School of Nursing, Faculty of Health Science, University of Namibia, Keetmanshoop, Namibia \\ ${ }^{2}$ School of Medicine, Faculty of Health Science, University of Namibia, Windhoek, Namibia \\ *Corresponding author E-mail: Inlukolo@unam.na
}

\begin{abstract}
Background: The benefits of antiretroviral therapy (ART) are undeniable: decreased morbidity and mortality, improved clinical outcomes, and decreased HIV transmission. In recent years, there have been remarkable efforts to roll out HIV support and treatment programs, resulting in ART access to nearly 4 million people in sub-Saharan Africa. However, a decisive step in improving outcomes of these programs relies on ART adherence. This study was conducted by aiming at exploring and describing the perceptions of patients on ART and treatment supporters about their roles in ART adherence. It was carried out at Oshakati ART clinic in the Intermediate Hospital Oshakati in Oshana region, Northern Namibia.

Methods: The qualitative investigation was carried out on patients on ART and treatment supporters by means of unstructured interviews through convenient sampling technique. The data were analysed by content analytical method.

Results: The study results from all respondents were consolidated, which gave a clear understanding regarding adherence at the ART clinic in Intermediate Hospital Oshakati. The perceptions of patients on ART and treatment supporters with regard to their role towards ART adherence were classified based on participants' experiences into two main themes and sub-themes, including: 1) ART patients' perceptions around their current roles in ART adherence comprise of four sub-themes of ART patients' perceptions about taking medication daily, perceptions about collection of medicine supply and follow-up visits, perceptions about food and medication and perceptions about support in ART treatment; 2) Treatment supporters' perceptions about their role in ART adherence consist of four sub-themes of perceptions about follow-up visits and collection of medicine, perceptions about psychosocial and emotional support, perceptions about giving food and medication and perceptions about encouraging personal hygiene.

Conclusion: Patients and treatment supporters perceived adherence as an important aspect in the success of antiretroviral treatment. Giving patients correct information, personal motivation, patients understanding of treatment, traditional and religious beliefs were among other factors perceived by treatment supporters to be impacting on ART adherence.
\end{abstract}

Keywords: Adherence; Anti-Retroviral Therapy (ART) ART Patients; HIV/AIDS; Treatment Supporters.

\section{Introduction}

The Acquired Immunodeficiency Syndrome (AIDS) is one of the most complex health problems of the $21^{\text {st }}$ century. Since its appearance in 1981, the HIV/AIDS pandemic, which leads to a serious challenge to humankind, without doubt, has become one of the most serious infectious diseases (Taher \& Abdelhai, 2011, p 57). Recent UNAIDS reports on the global AIDS epidemic estimates that there were 35.3 million (32.2- 38.8 million) people living with HIV. In 2012 worldwide, 2.3 million (1.9- 2.7 million people became newly infected with HIV, and 1.6 million (1.4 million-1.9 million) people died from AIDS-related causes (UNAIDS, 2013). The HIV epidemic has affected all parts of the globe; the level of severity has tended to vary depending on demographics of the country and the time when the virus was introduced throughout the region. Currently, the regions where the HIV has the most dramatic effects are sub-Saharan Africa, followed by Latin America and the Caribbean and Asia (Kristy et al, p.2).

Namibia is one of the five countries mostly affected by the world pandemic (MOHSS 2014, p. 11). Though the first case of HIV/AIDS in Namibia was reported in 1986, antiretroviral treatment (ART) was only introduced in 2003. In 2004, ART was piloted in 4 regions and in 2006 the service was rolled out to all districts and regional hospitals in the country, in order to make the service accessible to those who need them. Currently all three (3) intermediate hospitals, 34 district hospitals and 84 health centers provide ART services in Namibia. In Namibia, the national HIV/AIDS prevalence rate of 2008 was estimated to be $17,8 \%$, with a $21 \%$ rate for Oshana region. Oshana region is one of the 14 regions in Namibia, in the northern part of the country and in 2009, had an estimated population of 183452 for 2009. According to the Health Information System for Oshana region, 2008/2009, approximately 10009 HIV-positive patients were registered for antiretroviral therapy of which about $17 \%$ defaulted. This meant 
that $17 \%$ of the patients represented poor ART adherence (MOHSS, Health information report: 2009). By the end of 2005, about 14,400 patients were enrolled on ART programme in various public health facilities. In the 2008/2009 financial year, about 10009 HIV-positive patients were registered for ART at ART clinic in the Intermediate Hospital Oshakati, of which about 17\% defaulted from ART programme (National Census, 2011).

According to the National guidelines for Antiretroviral Therapy (2014, p.11), the recommended preferred First Line ART regimen for adults and adolescents with a CD4 of 500 cells $/ \mathrm{mm}$ and below, older than 10 years who weigh at least $35 \mathrm{~kg}$, all HIV infected pregnant and breast feeding women, is: tenofovir (TDF) + emtricitabine (FTC) or lamivudine (3TC) + efavirenz (EFV), given as a one fixed dose combination. Poor adherence may lead to failure of first line regimen which leads to patients being initiated on the less tolerated, more costly second line ART regimen: $\mathrm{Zi}$ dovudine (AZT), tenofovir (TDF), lamivudine (3ZT), and lopinavir (LPV/r).

The importance of adherence to ART treatment is that it helps the medicine to work effectively by suppressing the viral load and boosting the immune system of patients and decrease virus transmission. ART medicine adherence is vital for the success of Highly Active Antiretroviral Drugs, and very high levels of attendance, taking at least $95 \%$ of prescribed doses, is required to sustain suppression of HIV growth (MOHSS, 2007, p. 7).

Adherence to ART results in a decrease in viral load, morbidity and mortality. Adherence to ART improves survival, decreases hospitalization and acute illness and decreases the transmission of the virus. In a study done in Pittsburgh, USA, Paterson et al (2000, p. 20) also reported the importance of adherence in their study, patients with treatment adherence of $95 \%$ or greater had fewer hospitalization days than those with lower treatment adherence rates. In addition, no opportunistic infections or deaths occurred in patients who had an adherence rate of $95 \%$ or greater. Proper adherence also results in minimizing the emergence of drug-resistant strains and an increase in CD4 lymphocyte count as discussed below. Drug resistance is a burning issue that in turns to threaten the gains of ART treatment (Cahn, 2004, p.55).

The National guidelines for Antiretroviral Therapy (2014, p.16), recommended that for patients to initiate $A R V$, the must have treatment supporters; Treatment supporters must make sure they know much about ART treatment and clarify some of the myths and misconceptions of ART treatment. They must help health-care workers reinforce positive behavior among their patients and maximize ART adherence. Treatment supporters must also help patients by reminding them of their medication as well as taking their patients to ART clinic for follow ups and collection of medication; they also must offer psychosocial and emotional support to their patients.

HIV/AIDS is a common health problem and defaulters of antiretroviral treatment become a problem in Oshana region, and there is no study in the region that highlights the situation. It is against this background that the researcher intended to look at the perceptions of HIV-positive patients on ART, and treatment supporters about their roles related to ART adherence.

\section{Methodology}

The study is a qualitative, descriptive, exploratory and contextual in design as it was carried out through interviews, it was the most appropriate to provide information as needed. The study included HIV-positive patients on ART at Oshakati, Centre for disease Control (CDC) clinic and treatment supporters within Oshana region where Oshakati ART clinic falls.

\subsection{Sampling}

Convenient sampling was used in this study, to suit the researcher's busy schedule. The sample size was nine (9) HIV-positive patients on antiretroviral therapy, eleven (11) treatment supporters.

\subsection{Pilot study}

A pilot study was conducted with two (2) ART patients and two (2) treatment supporters in Intermediate Hospital Oshakati. It was carried out in order to test the practical aspects of the research study and to investigate the feasibility of the proposed study. All amendments were done before the actual study.

\subsection{Data collection}

This study utilized unstructured interview schedules to collect data from ART patients, treatment supporters. In-depth interviews with ART-patients and treatment supporters were conducted in a language of their choice, as the researcher is conversant with most of the languages spoken throughout the region. Particular care was taken to ensure that the respondents were comfortable and at ease prior to and during the interview. Probing was done to encourage them to talk freely about their perceptions about their roles toward ART adherence. The researcher took field notes during the interviews, and also tape recorded the entire interviews.

\subsection{Ethical considerations}

We obtained informed consent from the management, and from the HIV-positive patients selected from the interviews. The researcher assured confidentiality and the safe storage of information that was only accessible to authorized staff members such as the hospital superintendent, nurse manager and the researcher. The researcher assured that the subjects participated voluntarily and maintained a non-judgmental attitude toward the participants in the whole process (Bowling, 2002).

\subsection{Data analysis}

Content analysis was used to organize data into themes, categories and subcategories in order to synthesize valuable information and meaning from the respondents' raw data. The study results from all respondents were consolidated, which gave a clear understanding regarding adherence at ART clinic in Intermediate Hospital Oshakati.

\subsection{Demographic data of the participants}

\subsubsection{Patients on ART}

Responses were drawn from 4 male and 5 female HIV positive patients. The youngest among them was 25

Years of age and the oldest were 63 years of age. The average age of the patients was found to be 37.1 years.

These patients have on average been on the ART for 1.4 years. The shortest treatment period was 6 months while the longest period on ART was 25 months.

\subsubsection{Treatment supporters}

These are the people who are available most of the time to help and support patients, be it through the preparation of food, collection of medication, physiological support and many other ways. These patients were sons/ daughters, cousins, niece, nephew, or brothers of the supporters. Of importance, most of them were female and only one supporter was a male. This could be because females have the compassion to nurse other people compared to males. All the supporters engaged in the study were quite matured people as all of them were 30 years or older. On average, the patient supporters were aged 42 years. The period for which they have been supporting the patients' ranges from 1 year to 7 years. On average, they have been doing this support work for 3.3 years. 
These themes and sub-themes were generated and drawn up from the findings. The title chosen were those that seemed most logically related to the data (Table 2 and 3 )

Table 1: Summary of ART Patients' Perceptions around Their Current Roles in ART Adherence

\begin{tabular}{|c|c|c|c|}
\hline MAIN THEME & CATEGORY & SUB-CATEGORY & MEANING UNITS \\
\hline \multirow[t]{4}{*}{$\begin{array}{l}\text { ART patients' perceptions } \\
\text { around their roles in ART ad- } \\
\text { herence. }\end{array}$} & $\begin{array}{l}\text { ART patients' perceptions about } \\
\text { taking medication daily. }\end{array}$ & $\begin{array}{l}\text { Is a personal responsibility. } \\
\text { Done daily the same time. } \\
\text { Should not skip doses. }\end{array}$ & $\begin{array}{l}\text { To suppress the HIV virus. } \\
\text { To have enough medication in my } \\
\text { blood. } \\
\text { To avoid resistance. }\end{array}$ \\
\hline & $\begin{array}{l}\text { ART patients' perceptions about } \\
\text { collection of medicine supply and } \\
\text { follow up visits. }\end{array}$ & $\begin{array}{l}\text { Where ever you go carry enough } \\
\text { supply. }\end{array}$ & $\begin{array}{l}\text { To avoid missing doses which cause } \\
\text { resistance. Allow HCWs to review my } \\
\text { progress with treatment. } \\
\text { Make sure you always have your dos- } \\
\text { es. }\end{array}$ \\
\hline & $\begin{array}{l}\text { ART patients' perceptions about } \\
\text { food and medication. }\end{array}$ & $\begin{array}{l}\text { Must eat before you take medica- } \\
\text { tion. } \\
\text { You must avoid some foods and } \\
\text { alcohol. }\end{array}$ & $\begin{array}{l}\text { To avoid side effects as dizziness. } \\
\text { They make the medicine weak. }\end{array}$ \\
\hline & $\begin{array}{l}\text { ART patients' perceptions about } \\
\text { support in ART treatment. }\end{array}$ & $\begin{array}{l}\text { Must have treatment supporters. } \\
\text { Must work well with HCWs. }\end{array}$ & $\begin{array}{l}\text { Reminds on when to take medication } \\
\text { and cook food. } \\
\text { Give good information on counseling } \\
\text { and treatment. }\end{array}$ \\
\hline
\end{tabular}

\section{Results and discussions}

\subsection{ART patients' perceptions around their current roles in ART adherence}

\subsubsection{ART patients' perceptions about taking medication daily}

From the findings of this research, participants seem to have accepted their responsibility of taking medication daily. This category was further divided into sub-categories: Is a personal responsibility; done daily and at the same time; should not skip doses.

\subsubsection{Is a personal responsibility}

Most of the ART patients perceived taking medication daily as a personal responsibility. This was because of the realisation of the negative effects of not taking medication consistently as drug resistance and also the benefits of taking the medication consistently as increased CD4 count on follow up visits. The following are the quotations from the semi-in structure questionnaires:

"I take it as my responsibility for life and I feel good for it because there is a health benefit in it."

"I accept it as my responsibility for life to take medicine that rescued me from death, because I was seriously sick."

\subsubsection{Done daily at the same time}

Taking ART medicine daily at the same time was perceived as one of the pillars of effectiveness of ART treatment by ART patients who participate in this study. The reason given included the need to ensure that there is always enough medication in the blood to suppress the HIV virus. The following is an extract from the some of the responses from participants:

"I decided to join the program (ART) in order to drink medicine daily which will suppress the virus down in my blood and I will live longer, do my work as well".

\subsubsection{Should not skip doses}

Most of the respondents perceived not skipping doses as one of their responsibilities in ART adherence. Some of the respondents stressed that skipping doses would result in resistance to medication. They also expressed that resistance would make their bodies too weak to defend themselves and they will be bedridden. This they said would cause them to lose their income and would not be able to feed their children, according to one of the ART patients interviewed,

"Not skipping doses is good...I feel good that I am health and live longer, to help my children because they are young".

\subsubsection{ART patient's perceptions about collection of medication supplies and follow up visit?}

From the research findings, participants seemed to have accepted collection of medication supplies and going for follow up as their role in ART adherence. Most of the respondents emphasized that collection of medications and a follow up visit is of paramount importance in ART adherence. They said enough supply of medication ensures that no doses are missed which will ensure maximal viral suppression and eliminating drug resistance. They also expressed the benefits of follow up visits including being able to be clinically and immunologically evaluated by nurses and clinicians. It will also accord them the opportunity to be attended to any other illness as well as receive valuable reinforcement counselling. The following are some of the extracts from ART patients interviewed: "Though in the rain season flooding causes me to walk very long distance to the alternative bus stop...I try to come a day before so I will make time to come for follow up visit and collect my medication!"

\subsubsection{ART patient's perceptions about food and medication}

Most of the ART patients interviewed perceived eating healthily as one of their roles in ART adherence. All participants reported that they ate before they took their medication. To express the importance of food in ART treatment, some of the respondents expressed gratitude to relatives who bought or prepared food for them. Some of the respondents mentioned some of the adverse drug interactions which they perceived as occurring when they took their medication without food. One of the respondents cited dizziness as one of the side effects. Some of the quotations from extracts of participants' respondents are as follows:

"I eat first after 20 minutes I drink medicine. It helps to prevent dizziness."

"It could be good if government can source an organization that can provide food to patients on ARVs. I am just surviving in my disability grant and my mother's pension to fulfil all our needs including food."

\subsubsection{ART patient's perceptions about support in ART treat-} ment.

Having a treatment supporter is one of the qualifying factors in the initiation of patients on ART in Namibia though one can not be denied treatment in the absence of a treatment supporter. Most of the participants interviewed highlighted the importance of sourcing a treatment supporter as one of their perceived roles in ART adherence. Some of the ART patients expressed that it gave them strength to continue on ART when their families accepted them after knowing they were HIV positive. Most of the participants 
expressed that they created good relations with their close families who in return become their treatment supporters

Though a few said they did not want anyone to help them, this study revealed that treatment supporters are a valuable shoulder to cry on as well as someone to share laughter with. Some participants expressed that during the first two months of their treatment they experienced bouts of vomiting and diarrhea. They said that they felt like discontinuing treatment, but with the encouragement and counseling from their treatment supporters they continued. Some said that their treatment supporters collect their medications when they are not well enough to walk to the hospital or clinic to collect medicine and that they also cooking for them. Treatment supporters also provide psychosocial support. These perceptions of psychosocial support are expressed in some of the extracts from some structured interview as follows:
"The relationship with my mother and my sister is still good. They are my support in everything e.g. remind me and cook for me!"

"I had my sister already who is reminding me to take medicine. I need her to prepare food for me also. She is the one that put alarm for me, just to remind!"

"I do not think I need some body now, because I can look after myself. I have a reminder alarm for taking medicine and for follow-up as well."

\subsection{Treatment supporters' perceptions around their roles in ART adherence.}

Treatment supporters' perceptions around their roles in ART adherence were found to be positive and were divided into categories and sub-categories which are highlighted in table 3.

Table 2: Treatment Supporters' Perceptions around Their Roles in ART Adherence MAIN THEME
Treatment supporters' percep-
tions around their role in ART adherence

CATEGORY
Treatment supporters' perceptions
about follow up visits and collec-
about follow up visits and collection of medicine.

Treatment supporters' perceptions about psychosocial and emotional support.

Treatment supporters' perceptions about giving food and medication.

Treatment supporters' perceptions about encouraging personal hygiene.

SUB CATEGORY

Important huge responsibility.

Walking long distance for a purpose.

Initially drug reactions are worse.

Initially patients are weak and can't

walk or work.

Recovering can also be deceiving to the patient.

Too weak to remember to eat.

Must take medicine consistently every day.

HIV patients easily get opportunistic infections.

\section{MEANING UNITS}

To ensure adherence

health care workers measure our effort and gives us strength.

Need someone to encourage them to continue.

Need someone to talk to and do the all chores for them.

Some may want to fall back to their old habits.

Eating healthy help medicines to work and limit adverse effects.

To minimize drug resistance.

Personal hygiene minimizes communicable diseases.
The findings of the above categories and their sub-categories are presented below. Quotations in the participants own words have been used to enrich the findings of this research.

\subsubsection{Treatment supporters' perceptions about follow up visits and collections of medication}

From the findings of this research participants seem to have accepted their roles in ART adherence. Most of the treatment supporters perceived follow up visits and collection of medication as an important responsibility to maximize ART adherence as they have to make sure their patients attend all follow up visits without failure to minimize dose skipping and hence drug resistance. Treatment supporters perceived the hard task of walking long distances as a purpose driven task. Though they felt the pain of walking long distances, it was evident from their explanations that it was important for their patients to be reviewed by health-care workers. Clinical and immunological review of patients was a measure of the success of their efforts. If the patient improved after each follow-up visit, it would give them strength to continue. Like one treatment, supporter said, it clearly shows that the role of going with patients for follow-up is not an easy task as shown below.

"The long distance becomes worse during the rain season. It makes me to carry my son to pass through pools of water, but I notice the improvement of the health condition of my son...I am encouraged to continue to take care and walk $+45 \mathrm{~km}$ to reach the ART clinic at Intermediate Hospital Oshakati."

"We are footing sometimes because of lack of transport money."

"feel that to be responsible towards ART adherence..."

\subsubsection{Treatment supporter's perceptions about psychosocial and emotional support}

From the findings of the research, treatment supporters seem to have accepted their role of going for follow-up visits and collection of medication. However, they perceived it as a challenging task which they have evolved to cope with, with minimum hin- drances. Most of the participants echoed that during the initial phases of treatment, drug intolerance among patients is very high. A lot of the participants mentioned diarrhea and vomiting, rash, dizziness, headache and joint pain. These side effects of medication may be so severe that the patient may refuse to take their medication. So, those patients without supporters would have a high chance of defaulting. The treatment supporters also revealed how much valuable information they had obtained from formal training on drug interactions and side effects. They reported that at times the side effects are so severe that they had to take the patient to the doctor. The doctors changed medication for patients with severe side effects.

These sentiments are expressed in the following extracts from treatment supporter responses as follows:

"At the initiation of ARV syrup he starts vomiting and diarrhea for $5 / 12$ months, then doctor changed the syrup to tablets, currently he is gaining weight and he is no longer vomiting."

"The child developed skin rashes at the first month but now it disappeared."

"First month, the child (13 years) was vomiting and was always complaining that he cannot swallow the medicine well."

Most of the treatment supporters reported that at the initiation of ART, most of the patients are too weak to walk or work and as such cannot get to the ART clinic on their own. Without a treatment supporter, these patients would miss their follow-up visits as well as run out of medicines, and this would compromise ART adherence.

As one treatment supporter put it in response to the experiences gained as a treatment support,

"It gave me a burden to take care of her when she was weak initially, even during the night...I took her to the hospital, I feel good because she was sick and could not hear properly before ART treatment but now he is well."

Most of the treatment supporters expressed that when patients start to feel better they are encouraged to continue with treatment, but some become deceived and revert to their old negative habits. These negative patient habits may cause patients to default from their treatment. So treatment supporters perceived their role in 
psychosocial support as reinforcement of positive behavior to increase ART adherence. This was alluded to by one of the treatment supporters as follows:

"Some of my patients do not adhere to the treatment, or some continue to use alcohol, so I have to try to talk to them"

Some of the treatment supporters reported that they had to resort to consistently give health education to hence positive behavior on patients showing full signs of recovery.

\subsubsection{Treatment supporter's perceptions on giving food and medication on time}

Giving food and medication on time was perceived to be a critical role in ART adherence, by most treatment supporters. Almost all treatment supporters reported that preparing food was one of their chief responsibilities, as most of their patients were too weak to do house chores. Most of them reported that eating healthily every day before medication helps minimize drug intolerance. Forgetfulness to take medicine was one of the factors highlighted by treatment supporters as a hindrance to ART adherence. In order to minimize default due to forgetfulness some bought alarm clocks or programmed on their cell phones, the times for taking medication. This they say minimized skipping one of the requirements for taking ART medicine, and if not for treatment supporters, it would not be possible. Some of these sentiments are expressed below:

"To assist my cousin where needed, remind her to take her medication and her follow-up date in mind, support her psychologically and emotionally if needed."

"To give medicine at $07 \mathrm{H} 00$ and $19 \mathrm{H} 00$...prepare food for the child and lunch pack."

The treatment supporter caring for her nephew had this to say:

"To gives medicine on time 7H00 and 19H00 hours, and if we go on a visit, I carry the medication alone."

"To give him food in order to take medicine "

"Give medication...providing spiritual counseling, and to explain why they take medication."

\subsubsection{Treatment supporters' perceptions about encouraging personal hygiene}

Ensuring personal hygiene of patients was identified by some of the treatment supporters as a mainstay to prevent infectious diseases to which most HIV-positive patients are susceptible. HIVpositive patients are prone to opportunistic infections, which are easily picked up when personal hygiene is at a minimum.

"Make sure her clothes are clean, the environment...And encourage her to maintain personal hygiene."

"It is needed to take care of a patient's personal and environmental hygiene."

\section{Contributions}

This study is an original contribution to the body of knowledge on the perceptions of HIV-Positive patients on ART and Treatment Supporters with regard to their role towards ART adherence, at ART clinics in the Intermediate Hospital Oshakati, Namibia. The themes that were developed based upon the findings after analysis and interpretation of the results supported the aim of this study.

\section{Conclusions}

In this paper, the findings were presented. The two main themes identified for the perceptions of HIV-Positive patients on ART and Treatment Supporters with regard to their role towards ART adherence were discussed. Each of the main themes has several categories that were designed to further reduce the data into smaller manageable chunks. All two parties involved seem to understand their perceived roles as well as factors affecting ART adherence. Economic factors and lack of transport, money, poverty and distance to the clinic were some of the challenges identified by treatment supporters and ART patients interviewed in the study, which negatively affect ART adherence. Although distance to the clinic was perceived as a challenge by treatment supporters and ART patients, the study reported that these people were motivated by the benefits ART treatment gives them, and they now take the long distance as an opportunity to prolong life rather than a challenge. Overall, this study revealed that most of the participants perceived their roles in ART treatment positively and had a good knowledge base of factors affecting adherence.

\section{References}

[1] Arnsten SH, Demas PA, Farzadegan H, Grant RH, Sourevitch MN Chang CJ, Buono D, Eckholdt H, Howard AA \& Schoenbaum EE (2001) antiretroviral therapy adherence and viral suppression in HIV infected drug users: Comparison of self-report and electronic monitoring. Clinical infections Disease, 33: $1417-1423$ https://doi.org/10.1086/323201.

[2] Artlett JA, De Masi R, Quinn J, Moxham C \& Qousseau F ( 2001) Overview of the effectiveness of triple combination therapy in antiretroviral - naïve HIV-1 infected adults. AIDS, 15: 1369-1377. https://doi.org/10.1097/00002030-200107270-00006.

[3] Brink HI (1996) Fundamentals of research methodology for health care professionals. Cape Town: Creda Press.

[4] Bowling a (2002) Research method in health: Investigating Health and Health Services. Second edition: United States of America. Resource Centre. The Johns HopkinsUniversity. [Online], Available: http://baletthiv.com/page68.htm[accessed 7 July 2009].

[5] Clinical manual for management of the HIV infected adult (2005) Antiretroviral Therapy Adherence. Chapter 3. file://f:/ART. adherence/ARV search 5/getc.htm downloaded 04/11/2016.

[6] De Vos, AS \& Fouche CB (2000) General introduction to research design, data collection methods and data analysis in Research at grass roots: A primer for caring professions, edited by De Vos, AS. Pretoria: Van Schaik.

[7] Machtinger EL \& Bangsberg DR (2005) Adherence to HIV antiretroviral therapy. HIV insite knowledge Base Chapter. File://f:/ART adherence/insite.hmt downloaded 07/10/2016.

[8] Ministry of Health and Social Services (2007) National guideline for antiretroviral therapy. Republic of Namibia (second edition). Windhoek.

[9] Ministry of Health and Social Services (2008) ART Adherence Counseling Training for Health care Workers. Republic of Namibia, Windhoek.

[10] Ministry of Health and Social Services (2009) Integrated management of adolescent and adult Illness. Comprehensive HIV Care with $A R T$. Republic of Namibia, Windhoek

[11] Ministry of Health and Social Services (2008) Report of Windhoek 2008 National HIV Sentinel Survey. Republic of Namibia, Windhoek.

[12] Ministry of Health and Social Services (2014) National guidelines for Antiretroviral Therapy. Republic of Namibia,Windhoek

[13] Paterson DL, Swindells S, Motir J, Brester M, Vergis EN, Squier C, $\&$ Signh N ( 2000). Adherence to Protease inhibitor Therapy and outcomes in patients with HIV infection. Annals internal medicine, 133(1):21-30. https://doi.org/10.7326/0003-4819-133-1200007040-00004.

[14] Robson C (1993) Ch9-interview and questionnaire in Robson C really world Research. Blackwell, 227-243

[15] Taher E \& Abdelhai R (2011) Nurses knowledge, perceptions, and attitudes towards HIV/AIDS: Effect of a health education intervention on two nursing groups in Cairo University, Egypt. Journal of Public Health and Epidemiology, 3(4), 144-151).

[16] Turner BJ (2002) Adherence to antiretroviral therapy by human immonodefiency virus infected patients. The journal of infectious disease, $\quad 185 \quad$ supplements $2: 5143-5151$. https://doi.org/10.1086/340197.

[17] UNAIDS \& WHO (2007) Report on global AIDS epidemic. Executive summary, Geneva: Switzerland.

[18] UNAIDS (2012) Report on the Global AIDS Epidemic.

[19] http://www.unaids.org/en/media/unaids/contentassts/docments/epid emiology/2012/gr2012/20121120_UN AIDS_Global_Report_2012_en.pdf

[20] UNAIDS (2013) Report on the Global AIDS Epidemic. Retrieved from http://www.unaids.org/en/resources/campaign/globalreport2013/fac tsheet/. 